\title{
Identification of genes and pathways in the synovia of women with osteoarthritis by bioinformatics analysis
}

\author{
BOBIN MI ${ }^{1}$, GUOHUI LIU ${ }^{1}$, WU ZHOU ${ }^{1}$, HUIJUAN LV ${ }^{2}$, YI LIU ${ }^{1}$ and JING LIU ${ }^{1}$ \\ ${ }^{1}$ Department of Orthopaedics, Union Hospital, Tongji Medical College, Huazhong University of Science and Technology, \\ Wuhan, Hubei 430022; ${ }^{2}$ Department of Rheumatology, Tangdu Hospital, The Fourth Military Medical University, \\ Xi'an, Shaanxi 710000, P.R. China
}

Received September 12, 2017; Accepted December 5, 2017

DOI: $10.3892 / \mathrm{mmr} .2018 .8429$

\begin{abstract}
Osteoarthritis (OA) has a high prevalence in female patients and sex may be a key factor affecting the progression of OA. The aim of the present study was to identify genetic signatures in the synovial membranes of female patients with $\mathrm{OA}$ and to elucidate the potential associated molecular mechanisms. The gene expression profiles of the GSE55457 and GSE55584 datasets were obtained from the Gene Expression Omnibus database. Data of two synovial membranes from normal female individuals (GSM1337306 and GSM1337310) and two synovial membranes from female patients affected by OA (GSM1337327 and GSM1337330) were obtained from the dataset GSE55457, and those of three synovial membranes from female patients affected by OA (GSM1339628, GSM1339629 and GSM1339632) were obtained from the dataset GSE55584. Differentially expressed genes (DEGs) were identified by using Morpheus software. Protein-protein interaction (PPI) networks of the DEGs were constructed by using Cytoscape software. Subsequently, Gene Ontology (GO) function and Kyoto Encyclopaedia of Genes and Genomes (KEGG) pathway enrichment analyses of the top module of the PPI network were performed by using ClueGo. A total of 377 DEGs were identified in the synovial membranes of OA patients compared with those of normal individuals, including 164 upregulated and 213 downregulated genes. The top 10 hub
\end{abstract}

Correspondence to: Dr Guohui Liu or Dr Wu Zhou, Department of Orthopaedics, Union Hospital, Tongji Medical College, Huazhong University of Science and Technology, 1277 Jiefang Avenue, Wuhan, Hubei 430022, P.R. China

E-mail: liuguohui@medmail.com.cn

E-mail: 472976834@qq.com

Abbreviations: OA, osteoarthritis; GEO, Gene Expression Omnibus; IL-6, interleukin 6; GPR18, G protein-coupled receptor 18; DEGs, differentially expressed genes; PPI, protein-protein interaction; GO, Gene Ontology; KEGG, Kyoto Encyclopedia of Genes and Genomes

Key words: osteoarthritis, protein-protein interaction network analysis, synovium genes were ubiquitin (UB)C, ribosomal protein (RP) L23A, mammalian target of rapamycin, heat shock protein $90 \alpha$ family class A member 1, RPS28, RPL37A, RPS24, RPS4X, RPS18 and UBB. The results of the GO analysis indicated that the DEGs included in the top module of the PPI were mainly enriched in the terms 'nuclear-transcribed mRNA catabolic process', 'nonsense mediated decay', and 'cytoplasmic translation and ribosomal small subunit biogenesis'. KEGG pathway analysis indicated that the DEGs included in the top one module were mainly enriched in the 'ribosome' pathway. The present study provides a systematic, molecular-level understanding of the degeneration of the synovial membrane in the progression of OA in female patients. The hub genes and molecules associated with the synovial membrane may be used as biomarkers and therapeutic targets for the treatment of $\mathrm{OA}$ in female patients with OA.

\section{Introduction}

Osteoarthritis (OA) is a degenerative joint disease characterized by the erosion of articular cartilage and subchondral bone and inflammation of the synovial membrane $(1,2)$. The etiology of $\mathrm{OA}$ is affected by factors including aging, injury, obesity and heredity. OA currently affects $\sim 25 \%$ of the population aged $>18$ years and is expected to be the greatest cause of disability among patients aged $>40$ years $(3,4)$.

Recent studies have increasingly recognized the importance of the synovial membrane in the progression of OA (5). The synovial membrane is significantly altered prior to the occurrence of visible cartilage degeneration (6). Prieto-Potin et al reported that the histological pattern of the synovial membrane in OA patients was characterized by hyperplasia of synovial lining and stromal vascularization (7). Inflammatory cells (including macrophages, as well as B- and T-lymphocytes) and cytokines [including interleukin (IL)-6, IL-1 $\beta$ and tumor necrosis factor- $\alpha]$ have been detected in the synovial membranes of OA patients (8-10). It has been demonstrated that the synovium is associated with pain and poor functioning of the knee (11). However, the detailed pathogenic mechanisms in the synovia in the progression of OA have remained poorly understood and there is currently no available intervention strategy to slow down disease progression in patients with $\mathrm{OA}$. 
Microarray technology has markedly expanded the capacity of investigators to examine the pathogenic processes of various diseases $(12,13)$. This technology is an important tool for current functional genomic research. Numerous studies have used microarray technology to identify certain OA-specific proteins, including activating transcription factor $4, \mathrm{G}$ protein-coupled receptor 18, S100 calcium-binding protein A9/A8, prostaglandin D2 synthase and stromal cell-derived factor $1(14,15)$. However, the sex of a patient may have a key role in the development of OA: Epidemiological studies have revealed a higher incidence of OA in females than in males $(16,17)$. Furthermore, the use of certain medicines, including non-steroidal anti-inflammatory drugs or methotrexate, may also affect gene expression in synovial membranes affected by OA. In the present study, to exclude the effects of sex and medication use on the synovial membrane, genetic expression data of samples from female patients with OA who did not take any medicines and from healthy females were extracted. A bioinformatics analysis was performed to explore key genes whose functions are important in the synovial membranes of female patients with OA. Subsequently, Gene Ontology (GO) and Kyoto Encyclopedia of Genes and Genomes (KEGG) pathway enrichment analyses were performed, with the aim of gaining a better understanding of the molecular mechanisms of the synovia in female patients with OA.

\section{Materials and methods}

Microarray data. The Gene Expression Ominibus (GEO; https://www.ncbi.nlm.nih.gov/geo/) stores original submitter-supplied records as well as curated DataSets. Two gene expression profiles (GSE55457 and GSE55584) were obtained from the GEO database. To eliminate the potential effect of sex and medication, only data of synovial membranes from healthy females and female patients with OA who did not take any medicines were used. Data of two normal synovial membranes (GSM1337306 and GSM1337310) and two synovial membranes affected by OA (GSM1337327 and GSM1337330) were obtained from the GSE55457 dataset, while the data of three synovial membranes affected by OA (GSM1339628, GSM1339629 and GSM1339632) were obtained from the GSE55584 dataset.

Identification of differentially expressed genes (DEGS). Analysis was performed by using the matrix visualization and analysis platform Morpheus (https://software.broadinstitute.org/morpheus/). The expression of mRNAs with a signal-to-noise ratio of $>2$ or $<-2$ were defined as DEGs (18).

Protein-protein interaction (PPI) network analysis. To understand the functional interactions between these DEGs, a PPI network was constructed by using the web-based tool STRING (http://www.string-db.org). Subsequently, the PPI network was visualized by using Cytoscape software (http://www. cytoscape.org/). The top ten nodes ranked by the degree of interactions in the PPI network were selected. Subsequently, the plug-in Molecular Complex Detection (MCODE) was used to screen modules of the PPI network in Cytoscape. The selection criteria were set as follows: MCODE scores $\geq 3$ and number of nodes $\geq 3$.
Table I. Top ten genes with the highest degree of interaction in the protein-protein interaction network.

\begin{tabular}{lc} 
Gene & Degree of interaction \\
\hline UBC & 97 \\
RPL23A & 20 \\
MTOR & 20 \\
HSP90AA1 & 20 \\
RPS28 & 17 \\
RPL37A & 16 \\
RPS24 & 15 \\
RPS4X & 15 \\
RPS18 & 14 \\
UBB & 13
\end{tabular}

All of the top ten genes were downregulated in the protein-protein interaction network. UBC, ubiquitin C; RPL23A, ribosomal protein L23a; MTOR, mechanistic target of rapamycin; HSP90AA1, heat shock protein $90 \alpha$ family class A member 1; RPS28, ribosomal protein S28; RPL37A, ribosomal protein 37a; RPS24, ribosomal protein S24; RPS4X, ribosomal protein S4 X-Linked; RPS18, ribosomal protein 18 ; UBB, ubiquitin B.

GO and pathway enrichment analysis. In order to analyse the functions and pathways that the DEGs in the top module from the PPI network are involved in, GO and KEGG pathway enrichment analyses were performed using ClueGo (19). A cut-off of 0.4 was set for kappa score and terms including at least 3 genes were retrieved.

\section{Results}

Identification of DEGs. A total of 5 OA samples and 2 normal synovial samples were analyzed from the GSE55457 and GSE55584 datasets. The gene expression profiles were analyzed with Morpheus software. This analysis identified 164 up- and 213 downregulated DEGs in the OA samples compared with the normal samples. An expression heat map of the top 30 up- and downregulated DEGs is presented in Fig. 1.

PPI network construction and module analysis. Based on the information in the STRING and Cytoscape databases, the top 10 hub nodes with the highest degree of interaction were screened. These hub genes were ubiquitin (UB) $\mathrm{C}$, ribosomal protein (RP) L23A, mammalian target of rapamycin (mTOR), heat shock protein $90 \alpha$ family class A member 1 (HSP90AA1), RPS28, RPL37A, RPS24, RPS4X, RPS18 and UBB (Table I). Among these genes, UBC had the highest degree of interaction with 97 nodes. Six modules from the PPI network satisfied the criteria of MCODE scores $\geq 3$ and number of nodes $\geq 3$ (Table II). The results of the GO analysis indicated that in the category 'biological process' (BP), the DEGs included in the top module (Fig. 2) were significantly enriched in the GO terms 'nuclear-transcribed mRNA catabolic process', 'nonsense mediated decay' (GO:000184), 'cytoplasmic translation' (GO:0002181) and 'ribosomal small subunit biogenesis' (GO:0042274) (Fig. 3). In the GO category 


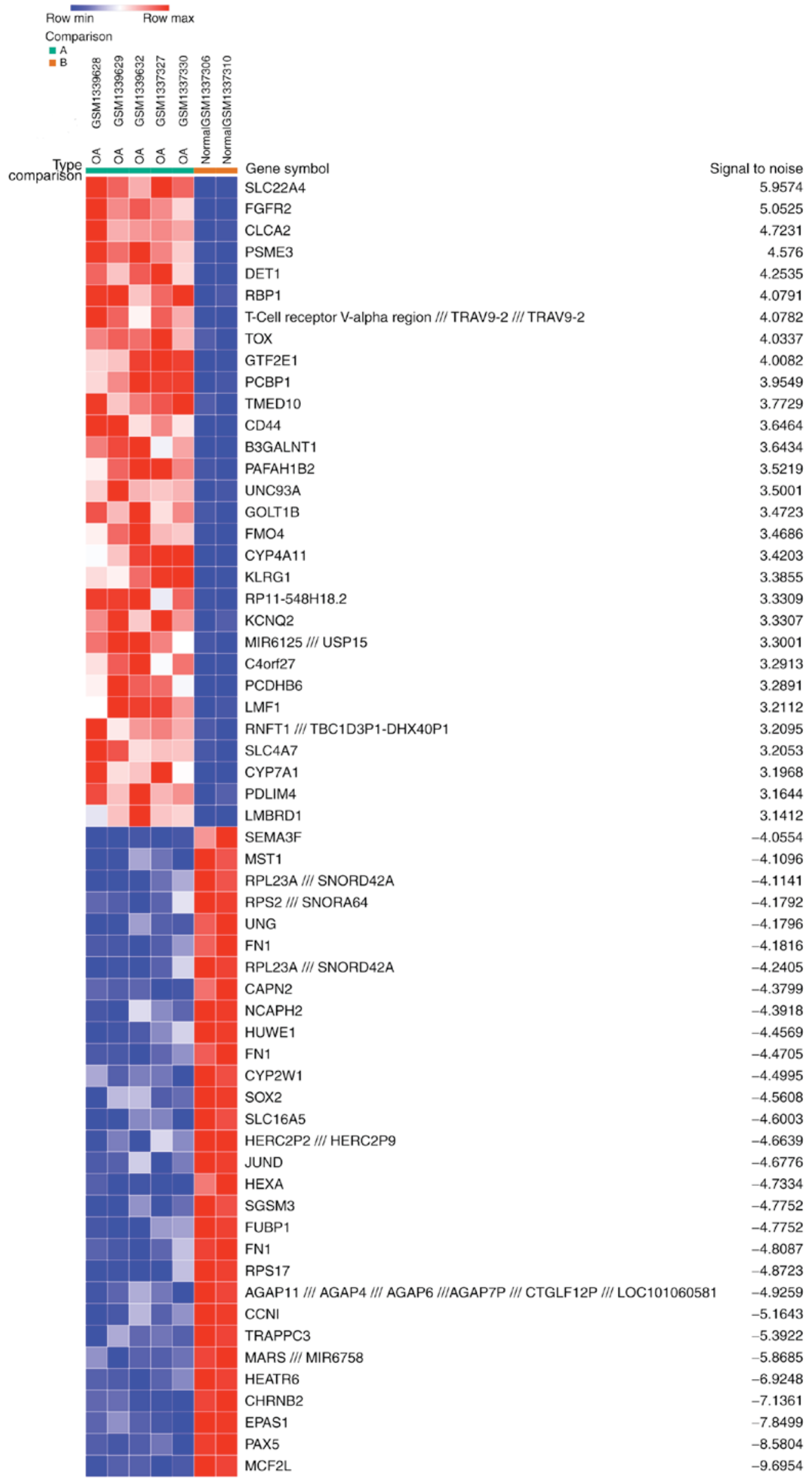

Figure 1. Heat map of the top 60 differentially expressed genes in patients with OA compared with those in a normal cohort ( 30 upregulated and 30 downregulated genes). Red, up-regulation; blue, down-regulation. Max, maximum; min, minimum; OA, osteoarthritis. 
Table II. Six modules from the protein-protein interaction network.

\begin{tabular}{lccrl}
\hline Cluster no. & Score & Nodes $(\mathrm{n})$ & Edges $(\mathrm{n})$ & \multicolumn{1}{c}{ Node IDs } \\
\hline 1 & 16.5 & 17 & 132 & $\begin{array}{l}\text { RPL19, RPS17, RPL23A, EEF1A1, RPS24, UBC, RPLP1, RPL37A, RPL22, } \\
\text { RPLP2, RPL14, RPS4X, RPS28, RPS18, EEF2, PABPC1, UBB }\end{array}$ \\
2 & 4 & 10 & 18 & $\begin{array}{l}\text { ATG14, CSNK1 A1, CHEK1, BECN1, MTOR, CSNK1E, CFLAR, GAPDH, } \\
3\end{array}$ \\
4 & 3 & 3 & 3 & YWHAB, UVRAG \\
5 & 3 & 3 & 3 & BUB3, CLASP2, KIF18A \\
6 & 3 & 3 & 3 & SEC31B, COPB1, ARF4 \\
& 3 & 3 & 3 & FPR1, PTGER3, GRM3
\end{tabular}

UBC, ubiquitin C; UBB, ubiquitin B; RPLP1, ribosomal protein lateral stalk subunit P1; RPLP2, ribosomal protein lateral stalk subunit P2; PABPC1, polyadenylate-binding protein; RPS28, ribosomal protein 28; RPS4X, ribosomal protein S4 X-Linked; RPS24, ribosomal protein S24; RPS17, ribosomal protein S17; RPS18, ribosomal protein S18; RPL23A, ribosomal protein L23a; RPL14, ribosomal protein L14; RPL19, ribosomal protein L19; RPL37A, ribosomal protein L37a; RPL22, ribosomal protein L22; EEF1A1, elongation factor 1- $\alpha$ 1; EEF2, eukaryotic translation elongation factor 2; ATG14, beclin 1-associated autophagy-related key regulator; CSNK1A1, casein kinase 1 $\alpha$ 1; CHEK1, checkpoint kinase 1; BECN1, beclin 1; MTOR, mechanistic target of rapamycin; CSNK1E, casein kinase 1 epsilon; CFLAR, CASP8 and FADD like apoptosis regulator; YWHAB, tyrosine 3-monooxygenase/tyrosine 5-monooxygenase activation protein $\beta$; UVRAG, UV radiation resistance associated gene; PCBP1, poly(rC) binding protein 1; SNRPD1, small nuclear ribonucleoprotein D1 polypeptide; HNRNPUL1, heterogeneous nuclear ribonucleoprotein U like 1; BUB3, budding uninhibited by benzimidazoles 3; CLASP2, cytoplasmic linker associated protein 2; KIF18A, kinesin family member 18 A; COPB1, coatomer protein complex subunit $\beta$ 1; ARF4, ADP ribosylation factor 4; FPR1, formyl peptide receptor 1; PTGER3, prostaglandin E receptor 3; GRM3, glutamate metabotropic receptor 3.

'molecular function' (MF), the DEGs included in the top module were significantly enriched in 'ribosomal (r)RNA binding' (GO:0019843) (Table III). The KEGG pathway enrichment analysis of the DEGs included in the top module revealed that these genes were mainly associated with the ribosome pathway (KEGG:03010) (Table III).

\section{Discussion}

In the present study, the DEGs and associated PPIs in the synovial membranes of female patients with OA compared with those in healthy females were investigated. These results may contribute to a better understanding of the etiology of the synovial membrane in $\mathrm{OA}$ in female patients. In addition, the identified hub genes may act as potential candidate biomarkers for the diagnosis of OA. As presented in Table I, the top 10 hub genes in the PPI network included UBC, RPL23A, mTOR, HSP90AA1, RPS28, RPL37A, RPS24, RPS4X, RPS18 and UBB. All hub genes were downregulated in the synovia of female patients with OA.

UBC and UBB are genes encoding ubiquitin in the mammalian genome. UB is a small protein that is involved in the maintenance of chromatin structure and the regulation of gene expression. Based on previous studies reporting that UB is essential for the growth of cancer cells $(20,21)$, the present study hypothesized that the upregulation of UBB and UBC may be a therapeutic strategy for maintaining the normal function of cells in the synovium.

mTOR is a major repressor of autophagy and it was previously demonstrated that mTOR is overexpressed in cartilage affected by OA (22). By inhibiting mTOR, authophagy is activated, which reduces cartilage damage and synovial inflammation in an experimental model of OA $(22,23)$. The present bioinformatics analysis indicated that mTOR was

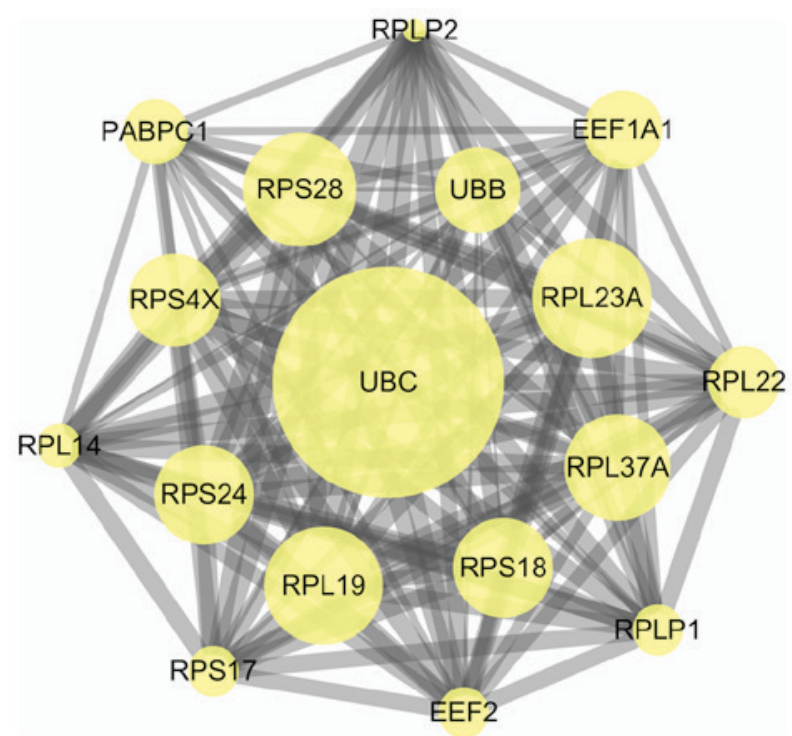

Figure 2. Top module from the protein-protein interaction network. UBC, ubiquitin C; UBB, ubiquitin B; RPLP1, ribosomal protein lateral stalk subunit P1; RPLP2, ribosomal protein lateral stalk subunit P2; PABPC1, polyadenylate-binding protein; RPS28, ribosomal protein 28; RPS4X, ribosomal protein S4, X-Linked; RPS24, ribosomal protein S24; RPS17, ribosomal protein S17; RPS18, ribosomal protein S18; RPL23A, ribosomal protein L23a; RPL14, ribosomal protein L14; RPL19, ribosomal protein L19; RPL37A, ribosomal protein L37a; RPL22, ribosomal protein L22; EEF1A1, elongation factor 1- $\alpha 1 ; \mathrm{EEF} 2$, eukaryotic translation elongation factor 2.

downregulated in the synovium of OA patients, suggesting that autophagy was activated in the early stage of OA.

RPs are the major constituents of the ribosome complex and their functions are essential for cell growth, proliferation and homeostasis. RPL23A has been detected in the cytoplasm of synovial cells from patients with rheumatoid arthritis (RA) or 
Table III. Analysis of GO terms in the category MF and KEGG pathways in the top module in the protein-protein interaction network.

\begin{tabular}{llll} 
Term & GO ID & \multicolumn{1}{c}{ Function } & \multicolumn{1}{c}{ Associated genes } \\
\hline MF & GO:0019843 & rRNA binding & EEF2, RPL19, RPL23A, RPS18, RPS4X \\
KEGG pathway & GO:0003010 & Ribosome & RPL14, RPL19, RPL22, RPL23A, RPL37A, RPLP1, RPLP2, RPS17, \\
& & & RPS18, RPS24, RPS28, RPS4X
\end{tabular}

MF, molecular function; GO, gene ontology; KEGG, Kyoto Encyclopedia of Genes and Genomes; rRNA, ribosomal RNA; RPLP1, ribosomal protein lateral stalk subunit P1; RPLP2, ribosomal protein lateral stalk subunit P2; RPS28, ribosomal protein 28; RPS4X, ribosomal protein S4 X-Linked; RPS24, ribosomal protein S24; RPS17, ribosomal protein S17; RPS18, ribosomal protein S18; RPL23A, ribosomal protein L23a; RPL37A, ribosomal protein L37a; RPL14, ribosomal protein L14; RPL19, ribosomal protein L19; RPL22, ribosomal protein L22; EEF2, eukaryotic translation elongation factor 2 .

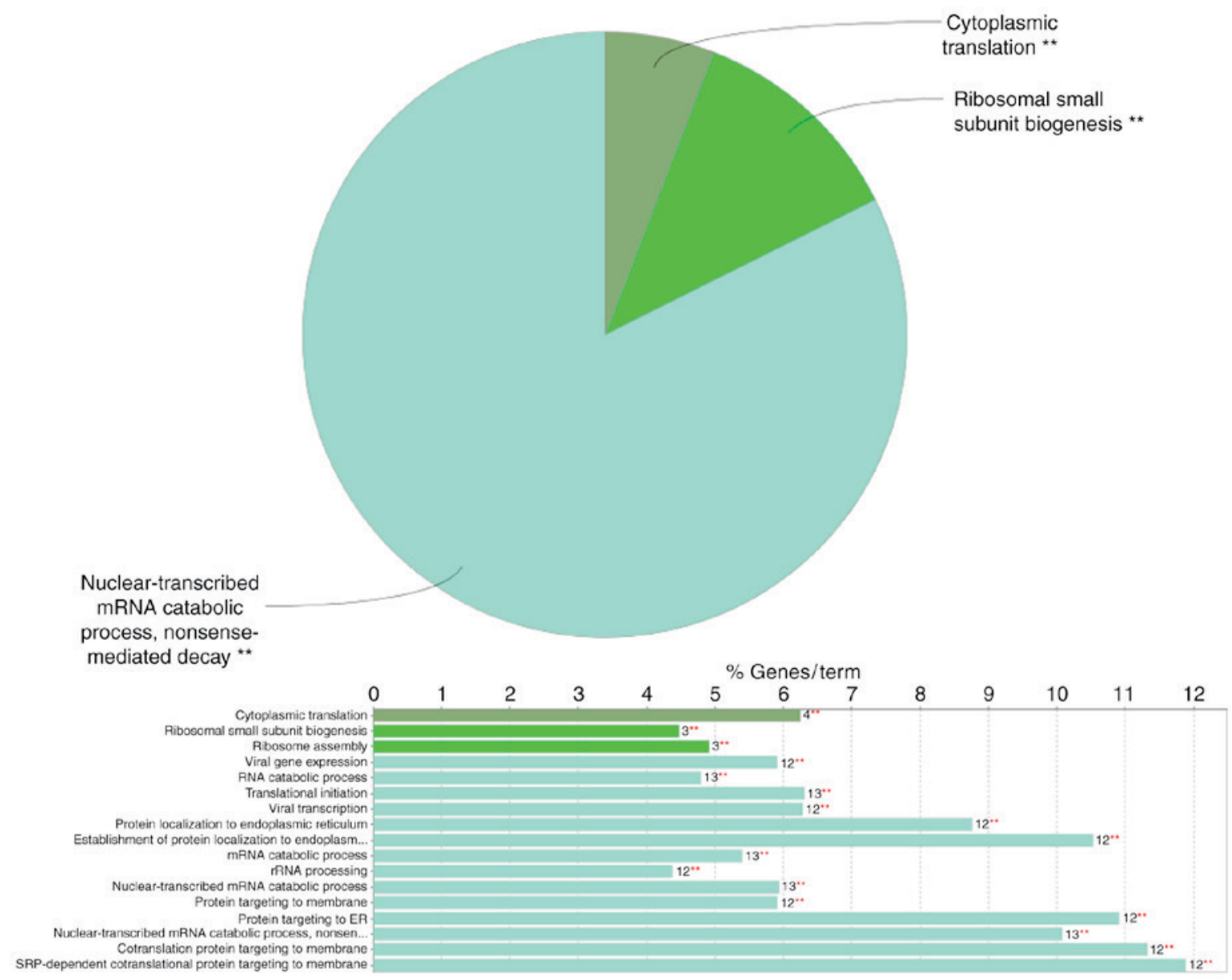

Figure 3. Functional distribution of Gene Ontology terms in the category 'biological processes' for the top module from the protein-protein interaction network. ${ }^{* *} \mathrm{P}<0.01$. rRNA, ribosomal RNA; ER, endoplasmic reticulum; SRP, signal recognition particle; number, associated genes with the biological function.

OA as well as in healthy individuals, and that in RA patients, RPL23A was attached to T cells (24). Downregulation of RPL23A may also cause abnormal functioning of the synovium in OA patients. Tang and Wade (25) reported that, in zebra finches, the total number of cells expressing RPL37 was lower in females than in males, and declined with increasing age in females. These results may explain why OA often occurs in older females. RPS24 insufficiency inhibits the proliferation of cancer cells (26) and causes distinct cell cycle defects in diamond-blackfan anemia (27). The biological functions of
RPS28, RPS18 and RPS4X in OA remain elusive at present. Anthony and Liebman (28) reported that alterations in RPS-18 may affect the translational accuracy in Saccharomyces cerevisiae. Furthermore, studies suggested that RPS28, RPS18 and RPS4X are associated with Diamond-Blackfan anemia (29-31). Based on these previous studies, it may be speculated that decreased levels of RPS28, RPS18 and RPS4X in synovial membranes may affect the functions of key proteins in rRNA processing, which may result in the degeneration of the synovium. 
The HSP90AA1 gene encodes the Hsp90 $\alpha$ protein. At present, the relevance of its expression to the progression of OA remains largely elusive. In a previous study, the upregulated expression of HSP90AA1 was demonstrated to be significantly associated with tumors and was critical for the stability of proteins that are vital for tumor progression (32). This result indicated that the level of HSP90AA1 downregulation may have an effect on cell viability in the synovium.

The ClueGo analysis provided the GO terms in the categories $\mathrm{BP}$ and $\mathrm{MF}$, as well as KEGG pathway terms associated with the DEGs included in the top module. With regard to BP terms, the DEGs included in the top module of the PPI network were mainly enriched in 'nuclear-transcribed mRNA catabolic process', 'nonsense mediated decay', 'cytoplasmic translation' and 'ribosomal small subunit biogenesis', which may otherwise lead to the synthesis of dysfunctional proteins (33). The other two enriched BP terms, 'cytoplasmic translation' and 'ribosomal small subunit biogenesis', have important roles in protein formation. In the GO category MF, the DEGs from the top module of the PPI network were enriched in the term 'rRNA binding'. KEGG pathway enrichment analysis indicated a significant role of 'ribosome' pathways in the degeneration of the synovial membrane.

As the association of most of the genes that were identified to be associated with OA in the present study were not previously reported, a further study will be performed to verify the gene expression levels of the screened genes in synovial samples from female patients with OA and healthy patients. Furthermore, cells extracted from those samples will be cultured to identify the molecular mechanisms of OA associated with these genes. In addition, the progression of synovial membrane degradation will be assessed in gene knockout rat models to further verify the functions of these genes.

The limitation of the present study is that only two normal synovial membrane samples and five OA synovial membrane samples were included in the analysis, and inclusion of other samples may change the present results. It is therefore necessary to collect more synovial membrane samples from female patients with OA to detect the expression levels of significantly DEGs.

In conclusion, the present study describes the molecular mechanism that may be involved in the degeneration of synovial membranes in female patients with OA. These mechanisms include 'nuclear-transcribed mRNA catabolic process', 'cytoplasmic translation' and 'ribosomal small subunit biogenesis'. In addition, the present study reported the major hub genes that may be involved in the molecular mechanisms associated with the synovium in female patients with OA. Sex disparities should be taken into consideration when further molecular biological experiments on OA are performed.

\section{Acknowledgements}

The authors would like to thank Mr. Jintong Ji (Department of Orthopaedics, Union Hospital, Tongji Medical College, Huazhong University of Science and Technology, Wuhan, China) for his continuous support and language editing of this article. This research was supported by the Natural Science Foundation of Hubei Province (grant no. WJ2017Q025) and the Science and Technology Department of Hubei Province (grant no. 2016CFB303).

\section{References}

1. Liu W, He J, Lin R, Liang J and Luo Q: Differential proteomics of the synovial membrane between bilateral and unilateral knee osteoarthritis in surgeryinduced rabbit models. Mol Med Rep 14: 2243-2249, 2016.

2. Chen S, Fang XQ, Zhang JF, Ma Y, Tang XZ, Zhou ZJ, Wang JY, Qin A and Fan SW: Lycorine protects cartilage through suppressing the expression of matrix metalloprotenases in rat chondrocytes and in a mouse osteoarthritis model. Mol Med Rep 14: 3389-3396, 2016.

3. Helmick CG, Felson DT, Lawrence RC, Gabriel S, Hirsch R, Kwoh CK, Liang MH, Kremers HM, Mayes MD, Merkel PA, et al: Estimates of the prevalence of arthritis and other rheumatic conditions in the United States. Part I. Arthritis Rheum 58: 15-25, 2008.

4. Thomas E, Peat G and Croft P: Defining and mapping the person with osteoarthritis for population studies and public health. Rheumatology (Oxford) 53: 338-345, 2014.

5. Kubosch EJ, Lang GM, Fürst D, Kubosch DC, Izadpanah K, Rolauffs B, Südkamp NP and Schmal H: The potential for synovium-derived stem cells in cartilage repair. Curr Stem Cell Res Ther, Oct 2, 2017 (Epub ahead of print).

6. Mathiessen A and Conaghan PG. Synovitis in osteoarthritis: Current understanding with therapeutic implications. Arthritis Res Ther 19: 18, 2017.

7. Prieto-Potin I, Largo R, Roman-Blas JA, Herrero-Beaumont G and Walsh DA: Characterization of multinucleated giant cells in synovium and subchondral bone in knee osteoarthritis and rheumatoid arthritis. BMC Musculoskelet Disord 16: 226, 2015.

8. Deligne C,CasulliS, Pigenet A, Bougault C,Campillo-Gimenez L, Nourissat G, Berenbaum F, Elbim C and Houard X: Differential expression of interleukin-17 and interleukin-22 in inflamed and non-inflamed synovium from osteoarthritis patients. Osteoarthritis Cartilage 23: 1843-1852, 2015.

9. Kuca-Warnawin EH, Kurowska WJ, Radzikowska A Massalska MA, Burakowski T, Kontny E, Słowińska I, Gasik R and Maśliński W: Different expression of chemokines in rheumatoid arthritis and osteoarthritis bone marrow. Reumatologia 54: 51-53, 2016.

10. Takano S, Uchida K, Miyagi M, Inoue G, Fujimaki H, Aikawa J, Iwase D, Minatani A, Iwabuchi K and Takaso M: Nerve growth factor regulation by TNF- $\alpha$ and IL- $1 \beta$ in synovial macrophages and fibroblasts in osteoarthritic mice. J Immunol Res 2016: 5706359, 2016.

11. Salaffi F, Ciapetti A and Carotti M: The sources of pain in osteoarthritis: A pathophysiological review. Reumatismo 66: 57-71, 2014

12. Das DK, Ali T, Krampis K and Ogunwobi OO: Fibronectin and androgen receptor expression data in prostate cancer obtained from a RNA-sequencing bioinformatics analysis. Data Brief 11: 131-135, 2017.

13. Gong C, Sun S, Liu B, Wang J and Chen X: Identification of potential therapeutic target genes, key miRNAs and mechanisms in oral lichen planus by bioinformatics analysis. Arch Oral Biol 78: 122-128, 2017.

14. Ramos YF, Bos SD, Lakenberg N, Böhringer S, den Hollander WJ, Kloppenburg M, Slagboom PE and Meulenbelt I: Genes expressed in blood link osteoarthritis with apoptotic pathways. Ann Rheum Dis 73: 1844-1853, 2014.

15. Ungethuem U, Haeupl T, Witt H, Koczan D, Krenn V, Huber H, von Helversen TM, Drungowski M, Seyfert C, Zacher J, et al: Molecular signatures and new candidates to target the pathogenesis of rheumatoid arthritis. Physiol Genomics 42A: 267-282, 2010.

16. Blagojevic M, Jinks C, Jeffery A and Jordan KP: Risk factors for onset of osteoarthritis of the knee in older adults: A systematic review and meta-analysis. Osteoarthritis Cartilage 18: 24-33, 2010.

17. Kee CC: Osteoarthritis: Manageable scourge of aging. Nurs Clin North Am 35: 199-208, 2000.

18. Venet D, Detours V and Bersini H: A measure of the signal-to-noise ratio of microarray samples and studies using gene correlations. PLoS One 7: e51013, 2012.

19. Bindea G, Mlecnik B, Hackl H, Charoentong P, Tosolini M, Kirilovsky A, Fridman WH, Pagès F, Trajanoski Z and Galon J: ClueGO: A Cytoscape plug-in to decipher functionally grouped gene ontology and pathway annotation networks. Bioinformatics 25: 1091-1032, 2009 . 
20. Oh C, Park S, Lee EK and Yoo YJ: Downregulation of ubiquitin level via knockdown of polyubiquitin gene Ubb as potential cancer therapeutic intervention. Sci Rep 3: 2623, 2013.

21. Tian Y, Ding W, Wang Y, Ji T, Sun S, Mo Q, Chen P, Fang Y, Liu J, Wang B, et al: Correction: Ubiquitin B in cervical cancer: Critical for the maintenance of cancer stem-like cell characters. PLoS One 11: e0152813, 2016.

22. Zhang Y, Vasheghani F, Li YH, Blati M, Simeone K, Fahmi H, Lussier B, Roughley P, Lagares D, Pelletier JP, et al: Cartilage-specific deletion of mTOR upregulates autophagy and protects mice from osteoarthritis. Ann Rheum Dis 74: 1432-1440, 2015.

23. Ribeiro M,López de Figueroa P, Nogueira-Recalde U, Centeno A, Mendes AF, Blanco FJ and Caramés B: Diabetes-accelerated experimental osteoarthritis is prevented by autophagy activation. Osteoarthritis Cartilage 24: 2116-2125, 2016.

24. Ito $Y$, Hashimoto M, Hirota K, Ohkura N, Morikawa H, Nishikawa H, Tanaka A, Furu M, Ito H, Fujii T, et al: Detection of $\mathrm{T}$ cell responses to a ubiquitous cellular protein in autoimmune disease. Science 346: 363-368, 2014.

25. Tang YP and Wade J: Sex- and age-related differences in ribosomal proteins L17 and L37, as well as androgen receptor protein in the song control system of zebra finches. Neuroscience 171: $1131-1140,2010$

26. Wang Y, Sui J, Li X, Cao F, He J, Yang B, Zhu X, Sun Y and $\mathrm{Pu}$ YD: RPS24 knockdown inhibits colorectal cancer cell migration and proliferation in vitro. Gene 571: 286-291, 2015.

27. Badhai J, Fröjmark AS, J Davey E, Schuster J and Dahl N: Ribosomal protein S19 and S24 insufficiency cause distinct cell cycle defects in Diamond-Blackfan anemia. Biochim Biophys Acta 1792: 1036-1042, 2009.

28. Anthony RA and Liebman SW: Alterations in ribosomal protein RPS28 can diversely affect translational accuracy in Saccharomyces cerevisiae. Genetics 140: 1247-1258, 1995.
29. Doherty L, Sheen MR, Vlachos A, Choesmel V, O'Donohue MF, Clinton C, Schneider HE, Sieff CA, Newburger PE, Ball SE, et al: Ribosomal protein genes RPS10 and RPS26 are commonly mutated in Diamond-Blackfan anemia. Am J Hum Genet 86: 222-228, 2010.

30. Gazda HT, Preti M, Sheen MR, O'Donohue MF, Vlachos A, Davies SM, Kattamis A, Doherty L, Landowski M, Buros C, et al: Frameshift mutation in p53 regulator RPL26 is associated with multiple physical abnormalities and a specific pre-ribosomal RNA processing defect in diamond-blackfan anemia. Hum Mutat 33: 1037-1044, 2012.

31. Gripp KW, Curry C, Olney AH, Sandoval C, Fisher J, Chong JX; UW Center for Mendelian Genomics, Pilchman L, Sahraoui R, Stabley DL and Sol-Church K: Diamond-Blackfan anemia with mandibulofacial dystostosis is heterogeneous, including the novel DBA genes TSR2 and RPS28. Am J Med Genet A 164A: 2240-2249, 2014.

32. Shen H, Zhu H, Song M, Tian Y, Huang Y, Zheng H, Cao R, Lin J, Bi Z and Zhong W: A selenosemicarbazone complex with copper efficiently down-regulates the $90-\mathrm{kDa}$ heat shock protein HSP90AA1 and its client proteins in cancer cells. BMC Cancer 14: 629, 2014.

33. Hentze MW and Kulozik AE: A perfect message: RNA surveillance and nonsense-mediated decay. Cell 96: 307-310, 1999.

This work is licensed under a Creative Commons Attribution-NonCommercial-NoDerivatives 4.0 International (CC BY-NC-ND 4.0) License. 